\title{
Exact Solutions with Variable Coefficient Function Forms for Conformable Fractional Partial Differential Equations by an Auxiliary Equation Method
}

\author{
Fanwei Meng $\mathbb{D}{ }^{1}$ and Qinghua Feng $\mathbb{D}^{2}$ \\ ${ }^{1}$ School of Mathematical Sciences, Qufu Normal University, Qufu, Shandong 273165, China \\ ${ }^{2}$ School of Mathematics and Statistics, Shandong University of Technology, Zibo, Shandong 255049, China \\ Correspondence should be addressed to Qinghua Feng; fqhua@sina.com
}

Received 22 March 2018; Accepted 29 July 2018; Published 5 August 2018

Academic Editor: Ziemowit Popowicz

Copyright (C) 2018 Fanwei Meng and Qinghua Feng. This is an open access article distributed under the Creative Commons Attribution License, which permits unrestricted use, distribution, and reproduction in any medium, provided the original work is properly cited.

\begin{abstract}
In this paper, an auxiliary equation method is introduced for seeking exact solutions expressed in variable coefficient function forms for fractional partial differential equations, where the concerned fractional derivative is defined by the conformable fractional derivative. By the use of certain fractional transformation, the fractional derivative in the equations can be converted into integer order case with respect to a new variable. As for applications, we apply this method to the time fractional two-dimensional Boussinesq equation and the space-time fractional (2+1)-dimensional breaking soliton equation. As a result, some exact solutions including variable coefficient function solutions as well as solitary wave solutions for the two equations are found.
\end{abstract}

\section{Introduction}

Fractional differential equations are the generalizations of classical differential equations with integer order derivatives. It is well known that fractional partial differential equations have proved to be very useful in many research fields such as physics, mathematical biology, engineering, fluid mechanics, plasma physics, optical fibers, neural physics, solid state physics, viscoelasticity, electromagnetism, electrochemistry, signal processing, control theory, chaos, finance, and fractal dynamics. In the last few decades, research on various aspects for fractional partial differential equations has received more and more attention by many authors, such as the oscillation $[1,2]$ and existence and uniqueness [3-5]. In order to better understand the physical process described by fractional partial differential equations, one usually needs to obtain exact solutions or numerical solutions for fractional partial differential equations. And so far a lot of effective methods have been developed and used by many authors. For example, these methods include the finite difference method [6], the $\left(G^{\prime} / G\right)$ method [7-10], the Jacobi elliptic function method [11], the projective Riccati equation method [12], the modified
Kudryashov method [13-19], the exp method [20-25], the ansatz method [26], the first integral method [27-29], and the subequation method [30-34]. Based on these methods, a lot of fractional partial differential equations have been investigated.

In this paper, we develop an auxiliary equation method for solving fractional partial differential equations, where the fractional derivative is defined in the sense of the conformable fractional derivative. Our aim is to seek exact solutions with variable coefficient function forms for some certain fractional partial differential equations.

The conformable fractional derivative is defined by [35]

$$
D_{t}^{\alpha} f(t)=\lim _{\varepsilon \rightarrow 0} \frac{f\left(t+\varepsilon t^{1-\alpha}\right)-f(t)}{\varepsilon} .
$$

The following properties for the conformable fractional derivative are well known, which can be easily proved due to the definition of the conformable fractional derivative.

(i) $D_{t}^{\alpha}[a f(t)+b g(t)]=a D^{\alpha} f(t)+b D^{\alpha} g(t)$.

(ii) $D_{t}^{\alpha}\left(t^{\gamma}\right)=\gamma t^{\gamma-\alpha}$. 
(iii) $D_{t}^{\alpha}[f(t) g(t)]=f(t) D^{\alpha} g(t)+g(t) D^{\alpha} f(t)$.

(iv) $D_{t}^{\alpha} C=0$, where $C$ is a constant.

(v) $D_{t}^{\alpha} f[g(t)]=f_{g}^{\prime}[g(t)] D_{t}^{\alpha} g(t)$.

(vi) $D_{t}^{\alpha}(f / g)(t)=\left(g(t) D^{\alpha} f(t)-f(t) D^{\alpha} g(t)\right) / g^{2}(t)$.

(vii) $D_{t}^{\alpha} f(t)=t^{1-\alpha} f^{\prime}(t)$.

The paper is organized as follows. In Section 2, we propose the description of the auxiliary equation method for seeking exact solutions with variable coefficient function forms for fractional partial differential equations. Then in Section 3, we apply the method to solve the time fractional two-dimensional Boussinesq equation and the space-time fractional $(2+1)$-dimensional breaking soliton equation. In Section 4, we present some concluding statements.

\section{Description of the Auxiliary Equation Method}

In this section, we give the description of the auxiliary equation method for solving fractional partial differential equations.

Suppose that a fractional partial differential equation in the independent variables $t, x_{1}, x_{2}, \ldots, x_{n}$ is given by

$$
P\left(u, u_{t}, u_{x_{1}}, \ldots, u_{x_{i}}, \ldots, D_{t}^{\alpha} u, D_{x_{1}}^{\gamma} u \ldots, D_{x_{i}}^{\beta} u, \ldots\right)=0,
$$

where $u$ is an unknown function, the orders of the fractional derivatives are, for example, $\alpha, \beta, \gamma \in(0,1]$, and $P$ is a polynomial in $u$ and its various partial derivatives including fractional derivatives. Without loss of generality, next we may assume that the fractional partial derivatives are related to the variables $t, x_{i}$, while the other variables are related to integer order derivatives.

Step 1. For those variables involving fractional derivatives, fulfil corresponding fractional transformations so that the fractional partial derivatives can be converted into integer order partial derivatives with respect to new variables.

Taking the expressions $D_{t}^{\alpha} u$ and $D_{x_{i}}^{\beta} u$, for example, one can use two fractional transformations $T=c\left(t^{\alpha} / \alpha\right)$ and $X_{i}=k\left(x_{i}^{\beta} / \beta\right)$ and denote $u\left(t, x_{1}, \ldots, x_{i}, \ldots, x_{n}\right)=$ $\widetilde{u}\left(T, x_{1}, \ldots, X_{i}, \ldots, x_{n}\right)$. Then due to the properties (ii) and $(v)$ of the conformable fractional derivative, one can obtain that $D_{t}^{\alpha} u=(\partial \tilde{u} / \partial T) D_{t}^{\alpha} T=(\partial \tilde{u} / \partial T) c=c \tilde{u}_{T}, D_{x_{i}}^{\beta} u=$ $\left(\partial \tilde{u} / \partial X_{i}\right) D_{x_{i}}^{\beta} X_{i}=\left(\partial \tilde{u} / \partial X_{i}\right) k=k \tilde{u}_{X_{i}}$. Therefore, the original fractional partial differential equation can be converted into another partial differential equation of integer order as follows:

$$
\widetilde{P}\left(\widetilde{u}, \tilde{u}_{T}, \tilde{u}_{x_{1}}, \ldots, \widetilde{u}_{X_{i}}, \ldots\right)=0 .
$$

Step 2. Suppose that the solution of (3) can be expressed by a polynomial in $\psi^{\prime}(\xi) / \psi(\xi)$ as follows:

$$
\begin{aligned}
\tilde{u} & \left(T, x_{1}, \ldots, X_{i}, \ldots, x_{n}\right) \\
& =\sum_{i=0}^{m} a_{i}\left(T, x_{1}, \ldots, X_{i}, \ldots, x_{n}\right)\left[\frac{\psi^{\prime}(\xi)}{\psi(\xi)}\right]^{i},
\end{aligned}
$$

where $\xi=\xi\left(T, x_{1}, \ldots, X_{i}, \ldots, x_{n}\right), a_{m}\left(T, x_{1}, \ldots, X_{i}, \ldots, x_{n}\right)$, $a_{m-1}\left(T, x_{1}, \ldots, X_{i}, \ldots, x_{n}\right), \ldots, a_{0}\left(T, x_{1}, \ldots, X_{i}, \ldots, x_{n}\right)$

are all unknown functions to be determined later with $a_{m}\left(T, x_{1}, \ldots, X_{i}, \ldots, x_{n}\right) \neq 0$, and $\psi=\psi(\xi)$ satisfies some certain auxiliary equation with the following form:

$$
F\left(\psi, \psi^{\prime}, \psi^{\prime \prime}, \ldots\right)=0
$$

whose solutions are known. Furthermore, as $\left[\left(\psi^{\prime}(\xi) /\right.\right.$ $\left.\psi(\xi))^{m}\right]^{\prime}=m\left(\psi^{\prime}(\xi) / \psi(\xi)\right)^{m-1}\left[\left(\psi(\xi) \psi^{\prime \prime}(\xi)-\left(\psi^{\prime}(\xi)\right)^{2}\right) / \psi^{2}(\xi)\right]$, then the degree of $\left[\psi^{\prime}(\xi) / \psi(\xi)\right]^{\prime}$ is usually one more than $\left[\psi^{\prime}(\xi) / \psi(\xi)\right]$. The positive integer $m$ can be determined by considering the homogeneous balance of the degrees between the highest order derivatives and nonlinear terms appearing in (3).

Step 3. Substituting (4) into (3), using the relation between $\psi^{\prime}(\xi)$ and $\psi(\xi)$ derived from (5), and collecting all terms with the same order of $\psi(\xi)$ together, the left-hand side of (3) is converted to another polynomial in $\psi(\xi)$. Equating each coefficient of this polynomial to zero yields a set of partial differential equations for $a_{m}\left(T, x_{1}, \ldots, X_{i}, \ldots\right.$, $\left.x_{n}\right), a_{m-1}\left(T, x_{1}, \ldots, X_{i}, \ldots, x_{n}\right) \ldots, \quad a_{0}\left(T, x_{1}, \ldots, X_{i}, \ldots, x_{n}\right)$, $\xi\left(T, x_{1}, \ldots, X_{i}, \ldots, x_{n}\right)$.

Step 4. Solving the equations yielded in Step 3 and using the solutions of (5), together with the fractional transformations introduced in Step 1, one can obtain exact solutions for (2).

Remark 1. In the present method, the expression of the transformation denoted by $\xi$ is underdetermined, and the coefficients $a_{i}$ in (4) are variable coefficient functions, which may contribute to the seeking of exact solutions with variable coefficient function forms. Furthermore, if (5) are selected for some different forms, such as the Riccati equation, Bernoulli equation, and Jacobi elliptic equation, then different exact solutions for (2) can be obtained correspondingly.

Remark 2. As the partial differential equations yielded in Step 3 are usually overdetermined, we may choose some special forms of $a_{m}, a_{m-1}, \ldots, a_{0}$ as in the following.

\section{Applications of the Auxiliary Equation Method}

3.1. Time Fractional Two-Dimensional Boussinesq Equation. We consider the time fractional two-dimensional Boussinesq equation with the following form:

$$
\begin{aligned}
D_{t}^{\alpha} D_{t}^{\alpha} u-u_{x x}-u_{y y}-\left(u^{2}\right)_{x x}-u_{x x x x}=0, & \\
& 0<\alpha \leq 1 .
\end{aligned}
$$

The fractional Boussinesq equation is used in the analysis of long waves in shallow water and also used in the analysis of many other physical applications, such as the percolation of water in a porous subsurface of a horizontal layer of material. Tasbozan et al. [36] solved (7) using the Jacobi elliptic function expansion method and obtained a series of exact solutions with Jacobi elliptic function forms. 
Now we use the proposed auxiliary equation method to solve (6). First we let $T=c\left(t^{\alpha} / \alpha\right)$ and $u(x, y, t)=\widetilde{u}(x, y, T)$. Then $D_{t}^{\alpha} u=c \widetilde{u}_{T}$, and (6) can be converted into the following form:

$$
c^{2} \tilde{u}_{T T}-\tilde{u}_{x x}-\tilde{u}_{y y}-\left(\tilde{u}^{2}\right)_{x x}-\tilde{u}_{x x x x}=0 .
$$

Suppose that the solution of (7) can be expressed by a polynomial in $\psi^{\prime}(\xi) / \psi(\xi)$ as follows:

$$
\tilde{u}(x, y, T)=\sum_{i=0}^{m} a_{i}(y, T)\left[\frac{\psi^{\prime}(\xi)}{\psi(\xi)}\right]^{i},
$$

where $a_{i}(y, T), i=0,1, \ldots, m$, are underdetermined functions and $\psi=\psi(\xi)$ satisfies (5). Balancing the degrees of $\tilde{u}_{x x x x}$ and $\left(\widetilde{u}^{2}\right)_{x x}$ in $(7)$, one can obtain $m+4=2 m+2$, which means $m=2$. Thus, one has

$$
\begin{aligned}
\tilde{u}(x, y, T)= & a_{2}(y, T)\left[\frac{\psi^{\prime}(\xi)}{\psi(\xi)}\right]^{2}+a_{1}(y, T)\left[\frac{\psi^{\prime}(\xi)}{\psi(\xi)}\right] \\
& +a_{0}(y, T) .
\end{aligned}
$$

Next we will discuss two cases, in which $\psi$ satisfies two certain auxiliary equations.

Case 1. $\psi=\psi(\xi)$ satisfies the following Riccati equation:

$$
\psi^{\prime}(\xi)+\lambda \psi(\xi)=\psi^{2}(\xi),
$$

where $\lambda \neq 0$.

Substituting (9) into (7), using (10), collecting all the terms with the same power of $\psi$ together, and equating each coefficient to zero yield a set of underdetermined partial differential equations $a_{0}(y, T), a_{1}(y, T), a_{2}(y, T)$, and $\xi(x, y, T)$. Solving these equations yields the following families of results, where $C_{1}, C_{2}$ are arbitrary constants and $F_{i}, i=$ $1,2, \ldots, 5$, are arbitrary functions.

Family 1

$$
\begin{aligned}
& a_{0}(y, T)=-\frac{1}{2} F_{1}(c y+T)^{2} \lambda^{2}-\frac{1}{2}, \\
& a_{1}(y, T)=-6 F_{1}(c y+T)^{2} \lambda, \\
& a_{2}(y, T)=-6 F_{1}(c y+T)^{2}, \\
& \xi(x, y, T)=F_{1}(c y+T) x+F_{2}(c y+T) .
\end{aligned}
$$

Family 2

$$
\begin{aligned}
& a_{0}(y, T)=F_{3}(-T-c y)+F_{4}(T-c y), \\
& a_{1}(y, T)=F_{2}(c y+T) \lambda, \\
& a_{2}(y, T)=F_{2}(c y+T), \\
& \xi(x, y, T)=F_{1}(c y+T) .
\end{aligned}
$$

Family 3

$$
\begin{aligned}
a_{0}(y, T) & =-\frac{1}{2} C_{1}^{2} \lambda^{2}-\frac{1}{2}, \\
a_{1}(y, T) & =-6 C_{1}^{2} \lambda, \\
a_{2}(y, T) & =-6 C_{1}^{2}, \\
\xi(x, y, T) & =C_{1} x+F_{1}(c y+T) .
\end{aligned}
$$

Family 4

$$
\begin{aligned}
& a_{0}(y, T)=\frac{-C_{1}^{2}+c^{2} C_{4}^{2}-C_{2}^{4} \lambda^{2}-C_{2}^{2}}{2 C_{2}^{2}}, \\
& a_{1}(y, T)=-6 C_{2}^{2} \lambda, \\
& a_{2}(y, T)=-6 C_{2}^{2}, \\
& \xi(x, y, T)=C_{1} y+C_{4} T+C_{2} x+C_{3} .
\end{aligned}
$$

Family 5

$$
\begin{aligned}
a_{0}(y, T) & =F_{4}(-T-c y)+F_{5}(T-c y), \\
a_{1}(y, T) & =F_{3}(c y+T), \\
a_{2}(y, T) & =F_{2}(c y+T), \\
\xi(x, y, T) & =F_{1}(c y+T) .
\end{aligned}
$$

Remark 3. It is obvious that if we let $F_{1}(c y+T)=k_{1}, F_{2}(c y+$ $T)=c y+T$ in Family 1, then the transformation denoted by $\xi$ becomes $\xi(x, y, T)=k_{1} x+c y+T=k_{1} x+c y+c\left(t^{\alpha} / \alpha\right)$, which has been used by many authors so far in the literature.

On the other hand, it is well known that the solution of (10) is denoted by

$$
\psi(\xi)=\frac{\lambda}{1+\lambda d e^{\lambda \xi}}
$$

where $d$ is an arbitrary constant, so

$$
\frac{\psi^{\prime}(\xi)}{\psi(\xi)}=-\frac{d \lambda^{2} e^{\lambda \xi}}{1+\lambda d e^{\lambda \xi}} .
$$

In particular, when $d=1 / \lambda$, one can obtain

$$
\frac{\psi^{\prime}(\xi)}{\psi(\xi)}=-\frac{\lambda}{2}\left[1+\tanh \left(\frac{\lambda \xi}{2}\right)\right] .
$$

By a combination of the results denoted in Families 1-5 and (17), together with the expression of $\xi$, one can obtain a series of exact solutions for the time fractional twodimensional Boussinesq equation as follows:

$$
\begin{aligned}
u_{1}(x, y, t)= & -6\left[F_{1}(c y+T)\right]^{2}\left(\frac{d \lambda^{2} e^{\lambda \xi}}{1+\lambda d e^{\lambda \xi}}\right)^{2} \\
& +6\left[F_{1}(c y+T)\right]^{2} \lambda\left(\frac{d \lambda^{2} e^{\lambda \xi}}{1+\lambda d e^{\lambda \xi}}\right) \\
& -\frac{1}{2}\left[F_{1}(c y+T)\right]^{2} \lambda^{2}-\frac{1}{2},
\end{aligned}
$$


where $T=c\left(t^{\alpha} / \alpha\right), \xi=F_{1}(c y+T) x+F_{2}(c y+T)$, and $F_{1}, F_{2}$ are arbitrary functions.

$$
\begin{aligned}
u_{2}(x, y, t)= & F_{2}(c y+T)\left(\frac{d \lambda^{2} e^{\lambda \xi}}{1+\lambda d e^{\lambda \xi}}\right)^{2} \\
& -F_{2}(c y+T) \lambda\left(\frac{d \lambda^{2} e^{\lambda \xi}}{1+\lambda d e^{\lambda \xi}}\right) \\
& +F_{3}(-T-c y)+F_{4}(T-c y),
\end{aligned}
$$

where $T=c\left(t^{\alpha} / \alpha\right), \xi=F_{1}(c y+T)$, and $F_{1}, F_{2}, F_{3}, F_{4}$ are arbitrary functions.

$$
\begin{aligned}
u_{3}(x, y, t)= & -6 C_{1}^{2}\left(\frac{d \lambda^{2} e^{\lambda \xi}}{1+\lambda d e^{\lambda \xi}}\right)^{2} \\
& +6 C_{1}^{2} \lambda\left(\frac{d \lambda^{2} e^{\lambda \xi}}{1+\lambda d e^{\lambda \xi}}\right)-\frac{1}{2} C_{1}^{2} \lambda^{2}-\frac{1}{2},
\end{aligned}
$$

where $T=c\left(t^{\alpha} / \alpha\right), \xi=C_{1} x+F_{1}(c y+T), C_{1}$ is an arbitrary constant, and $F_{1}$ is an arbitrary function.

$$
\begin{aligned}
u_{4}(x, y, t)= & -6 C_{2}^{2}\left(\frac{d \lambda^{2} e^{\lambda \xi}}{1+\lambda d e^{\lambda \xi}}\right)^{2} \\
& +6 C_{2}^{2} \lambda\left(\frac{d \lambda^{2} e^{\lambda \xi}}{1+\lambda d e^{\lambda \xi}}\right) \\
& +\frac{-C_{1}^{2}+c^{2} C_{4}^{2}-C_{2}^{4} \lambda^{2}-C_{2}^{2}}{2 C_{2}^{2}},
\end{aligned}
$$

where $T=c\left(t^{\alpha} / \alpha\right), \xi=C_{1} y+C_{4} T+C_{2} x+C_{3}$, and $C_{1}, C_{2}$, $C_{3}, C_{4}$ are arbitrary constants.

$$
\begin{aligned}
u_{5}(x, y, t)= & F_{2}(c y+T)\left(\frac{d \lambda^{2} e^{\lambda \xi}}{1+\lambda d e^{\lambda \xi}}\right)^{2} \\
& -F_{3}(c y+T)\left(\frac{d \lambda^{2} e^{\lambda \xi}}{1+\lambda d e^{\lambda \xi}}\right) \\
& +F_{4}(-T-c y)+F_{5}(T-c y),
\end{aligned}
$$

where $T=c\left(t^{\alpha} / \alpha\right), \xi=F_{1}(c y+T), F_{1}, F_{2}, F_{3}, F_{4}, F_{5}$ are arbitrary functions.

Similarly, by a combination of the results in Families 1-5 and (18), one can obtain the following solitary wave solutions, in which $T=c\left(t^{\alpha} / \alpha\right), C_{i}, i=1,2,3,4$, are arbitrary constants, and $F_{j}, j=1,2,3,4,5$, are arbitrary functions.

$$
\begin{aligned}
u_{6}(x, y, t)= & -\frac{3 \lambda^{2}}{2}\left[F_{1}(c y+T)\right]^{2}\left[1+\tanh \left(\frac{\lambda \xi}{2}\right)\right]^{2} \\
& +3\left[F_{1}(c y+T)\right]^{2} \lambda^{2}\left[1+\tanh \left(\frac{\lambda \xi}{2}\right)\right] \\
& -\frac{1}{2}\left[F_{1}(c y+T)\right]^{2} \lambda^{2}-\frac{1}{2}
\end{aligned}
$$

where $\xi=F_{1}(c y+T) x+F_{2}(c y+T)$.

$$
\begin{aligned}
u_{7}(x, y, t)= & \frac{\lambda^{2}}{4} F_{2}(c y+T)\left[1+\tanh \left(\frac{\lambda \xi}{2}\right)\right]^{2} \\
& -\frac{\lambda^{2}}{2} F_{2}(c y+T)\left[1+\tanh \left(\frac{\lambda \xi}{2}\right)\right] \\
& +F_{3}(-T-c y)+F_{4}(T-c y),
\end{aligned}
$$

where $\xi=F_{1}(c y+T)$.

$$
\begin{aligned}
u_{8}(x, y, t)= & -\frac{3 C_{1}^{2} \lambda^{2}}{2}\left[1+\tanh \left(\frac{\lambda \xi}{2}\right)\right]^{2} \\
& +3 C_{1}^{2} \lambda^{2}\left[1+\tanh \left(\frac{\lambda \xi}{2}\right)\right]-\frac{1}{2} C_{1}^{2} \lambda^{2} \\
& -\frac{1}{2}
\end{aligned}
$$

where $\xi=C_{1} x+F_{1}(c y+T)$.

$$
\begin{aligned}
u_{9}(x, y, t)= & -\frac{3 C_{2}^{2} \lambda^{2}}{2}\left[1+\tanh \left(\frac{\lambda \xi}{2}\right)\right]^{2} \\
& +3 C_{2}^{2} \lambda^{2}\left[1+\tanh \left(\frac{\lambda \xi}{2}\right)\right] \\
& +\frac{-C_{1}^{2}+c^{2} C_{4}^{2}-C_{2}^{4} \lambda^{2}-C_{2}^{2}}{2 C_{2}^{2}}
\end{aligned}
$$

where $\xi=C_{1} y+C_{4} T+C_{2} x+C_{3}$.

$$
\begin{aligned}
u_{10}(x, y, t)= & \frac{\lambda^{2}}{4} F_{2}(c y+T)\left[1+\tanh \left(\frac{\lambda \xi}{2}\right)\right]^{2} \\
& -\frac{\lambda}{2} F_{3}(c y+T)\left[1+\tanh \left(\frac{\lambda \xi}{2}\right)\right] \\
& +F_{4}(-T-c y)+F_{5}(T-c y),
\end{aligned}
$$

where $\xi=F_{1}(c y+T)$.

In Figure 1 , the solitary wave solution $u_{6}(x, y, t)$ in (24) with some special parameters is demonstrated.

Case 2. $\psi=\psi(\xi)$ satisfies the following equation:

$$
\psi^{\prime}+\lambda \psi=\mu \psi^{3}
$$

where $\lambda, \mu$ are arbitrary constants with $\lambda \neq 0$.

Substituting (9) into (7), using (29), collecting all the terms with the same power of $\psi$ together, and equating each coefficient to zero yield a set of underdetermined partial differential equations. Solving these equations yields the following results, where $C_{i}, i=1,2,3,4$, are arbitrary constants and $F_{i}, i=1,2,3,4$, are arbitrary functions. 


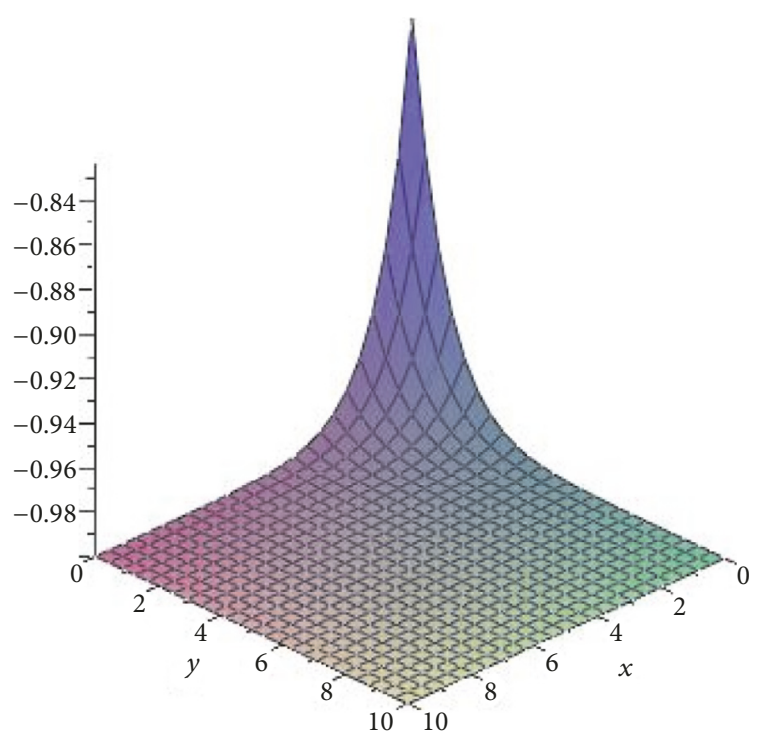

FIGURE 1: The solitary wave solution $u_{6}$ with $\alpha=0.5, F_{1}(c y+T)=1$, $F_{2}(c y+T)=c y+T, c=\lambda=1, t=3$.

Family 1

$$
\begin{aligned}
& a_{0}(y, T)=-2 F_{1}(c y+T)^{2} \lambda^{2}-\frac{1}{2}, \\
& a_{1}(y, T)=-24 F_{1}(c y+T)^{2} \lambda, \\
& a_{2}(y, T)=-24 F_{1}(c y+T)^{2}, \\
& \xi(x, y, T)=F_{1}(c y+T) x+F_{2}(c y+T) .
\end{aligned}
$$

Family 3

$$
\begin{aligned}
& a_{0}(y, T)=-2 C_{1}^{2} \lambda^{2}-\frac{1}{2}, \\
& a_{1}(y, T)=-24 C_{1}^{2} \lambda, \\
& a_{2}(y, T)=-24 C_{1}^{2}, \\
& \xi(x, y, T)=C_{1} x+F_{1}(c y+T) .
\end{aligned}
$$

\section{Family 4}

$$
\begin{aligned}
a_{0}(y, T) & =F_{3}(-T-c y)+F_{4}(T-c y), \\
a_{1}(y, T) & =F_{2}(c y+T) \lambda, \\
a_{2}(y, T) & =F_{2}(c y+T), \\
\xi(x, y, T) & =F_{1}(c y+T) .
\end{aligned}
$$

For the solutions of (29), one has

$$
\psi(\xi)= \pm \frac{1}{\sqrt{\mu / \lambda+A e^{2 \lambda \xi}}}
$$

where $A$ is an arbitrary constant and $\mu^{2}+A^{2} \neq 0$, so

$$
\frac{\psi^{\prime}(\xi)}{\psi(\xi)}=-\frac{A \lambda e^{2 \lambda \xi}}{\mu / \lambda+A e^{2 \lambda \xi}} .
$$

Substituting the results denoted by Families 1-4 into (9) and combining them with (35), one can obtain the following exact solutions for the time fractional two-dimensional Boussinesq equation, in which $T=c\left(t^{\alpha} / \alpha\right)$.

$$
\begin{aligned}
u_{11}(x, y, t)= & -24 F_{1}(c y+T)^{2}\left(\frac{A \lambda e^{2 \lambda \xi}}{\mu / \lambda+A e^{2 \lambda \xi}}\right)^{2} \\
& +24 F_{1}(c y+T)^{2} \lambda\left(\frac{A \lambda e^{2 \lambda \xi}}{\mu / \lambda+A e^{2 \lambda \xi}}\right) \\
& -2 F_{1}(c y+T)^{2} \lambda^{2}-\frac{1}{2}
\end{aligned}
$$

where $\xi=F_{1}(c y+T) x+F_{2}(c y+T)$.

$$
\begin{aligned}
u_{12}(x, y, t)= & -24 C_{2}^{2}\left(\frac{A \lambda e^{2 \lambda \xi}}{\mu / \lambda+A e^{2 \lambda \xi}}\right)^{2} \\
& +24 C_{2}^{2} \lambda\left(\frac{A \lambda e^{2 \lambda \xi}}{\mu / \lambda+A e^{2 \lambda \xi}}\right) \\
& +\frac{-C_{1}^{2}-4 C_{2}^{4} \lambda^{2}-C_{2}^{2}+c^{2} C_{4}^{2}}{2 C_{2}^{2}},
\end{aligned}
$$

where $\xi=C_{1} y+C_{4} T+C_{2} x+C_{3}$.

$$
\begin{aligned}
u_{13}(x, y, t)= & -24 C_{1}^{2}\left(\frac{A \lambda e^{2 \lambda \xi}}{\mu / \lambda+A e^{2 \lambda \xi}}\right)^{2} \\
& +24 C_{1}^{2} \lambda\left(\frac{A \lambda \mathrm{e}^{2 \lambda \xi}}{\mu / \lambda+A e^{2 \lambda \xi}}\right)-2 C_{1}^{2} \lambda^{2} \\
& -\frac{1}{2}
\end{aligned}
$$

where $\xi=C_{1} x+F_{1}(c y+T)$. 


$$
\begin{aligned}
u_{14}(x, y, t)= & F_{2}(c y+T)\left(\frac{A \lambda e^{2 \lambda \xi}}{\mu / \lambda+A e^{2 \lambda \xi}}\right)^{2} \\
& -F_{2}(c y+T) \lambda\left(\frac{A \lambda e^{2 \lambda \xi}}{\mu / \lambda+A e^{2 \lambda \xi}}\right) \\
& +F_{3}(-T-c y)+F_{4}(T-c y),
\end{aligned}
$$

where $\xi=F_{1}(c y+T)$.

If we set $\mu=\lambda A$ in (35), then we obtain the following solitary wave solutions:

$$
\frac{\psi^{\prime}(\xi)}{\psi(\xi)}=-\frac{\lambda}{2}[(1+\tanh (\lambda \xi))] .
$$

By a combination of the results in Families 1-4 and (40), one can obtain corresponding solitary wave solutions, which are omitted here.

3.2. Space-Time Fractional (2+1)-Dimensional Breaking Soliton Equation. We consider the space-time fractional $(2+1)$ dimensional breaking soliton equation [37] of the form

$$
\begin{gathered}
D_{t}^{\alpha} D_{x}^{\alpha} u-4\left(D_{x}^{\alpha} u\right)\left(D_{y}^{\alpha} D_{x}^{\alpha} u\right)-2\left(D_{x}^{\alpha} D_{x}^{\alpha} u\right) D_{y}^{\alpha} u \\
+D_{y}^{\alpha} D_{x}^{\alpha} D_{x}^{\alpha} D_{x}^{\alpha} u=0, \quad 0<\alpha \leq 1 .
\end{gathered}
$$

Now we solve (41) using the introduced auxiliary equation method.

Let $T=c\left(t^{\alpha} / \alpha\right), X=k_{1}\left(x^{\alpha} / \alpha\right), Y=k_{1}\left(y^{\alpha} / \alpha\right)$, and $u(x, y, t)=\tilde{u}(X, Y, T)$. Then $D_{t}^{\alpha} u=c \tilde{u}_{T}, D_{x}^{\alpha} u=k_{1} \tilde{u}_{X}$, $D_{y}^{\alpha} u=k_{2} \tilde{u}_{Y}$, and (41) can be converted into the following equation:

$$
\begin{aligned}
& c k_{1} \tilde{u}_{X T}-4 k_{1}^{2} k_{2} \widetilde{u}_{X} \widetilde{u}_{X Y}-2 k_{1}^{2} k_{2} \widetilde{u}_{X X} \widetilde{u}_{Y}+k_{1}^{3} k_{2} \tilde{u}_{X X X Y} \\
& \quad=0
\end{aligned}
$$

Suppose that the solutions of (42) can be expressed by a polynomial in $\psi^{\prime}(\xi) / \psi(\xi)$ as follows:

$$
\widetilde{u}(X, Y, T)=\sum_{i=0}^{m} a_{i}(Y, T)\left[\frac{\psi^{\prime}(\xi)}{\psi(\xi)}\right]^{i}
$$

where $a_{i}(Y, T)$ are underdetermined functions. Balancing the degrees of $\widetilde{u}_{X X X Y}$ and $\widetilde{u}_{X X} \widetilde{u}_{Y}$ in (42), one has $m=1$. Therefore,

$$
\tilde{u}(X, Y, T)=a_{1}(Y, T)\left[\frac{\psi^{\prime}(\xi)}{\psi(\xi)}\right]+a_{0}(Y, T) .
$$

Case 1. If $\psi(\xi)$ satisfies (10), then substituting (44) into (42), collecting all the terms with the same power of $\psi$ together, and equating each coefficient to zero yield a set of underdetermined partial differential equations for $a_{0}(Y, T)$, $a_{1}(Y, T), \xi(X, Y, T)$. Solving these equations yields several families of results as follows.
Family 1

$$
\begin{aligned}
a_{0}(Y, T) & =\frac{1}{2} C_{3} \lambda T+\frac{c C_{3}}{4 k_{2} C_{1} k_{1}} Y+C_{5}, \\
a_{1}(Y, T) & =C_{3} T+C_{4}, \\
\xi(X, Y, T) & =C_{1} X+C_{2} .
\end{aligned}
$$

Family 2

$$
\begin{aligned}
a_{0}(Y, T) & =\frac{1}{2} C_{1} k_{1} \lambda^{2} F(Y)+C_{2}, \\
a_{1}(Y, T) & =2 C_{1} k_{1}, \\
\xi(X, Y, T) & =C_{1} X+F(Y),
\end{aligned}
$$

where $F$ is an arbitrary function.

By a combination of (17), (44), and the results above, one can obtain the following exact solutions for the spacetime fractional $(2+1)$-dimensional breaking soliton equation, in which $T=c\left(t^{\alpha} / \alpha\right), X=k_{1}\left(x^{\alpha} / \alpha\right), Y=k_{1}\left(y^{\alpha} / \alpha\right)$.

$$
\begin{aligned}
u_{1}(x, y, t)= & -\left(C_{3} T+C_{4}\right)\left(\frac{d \lambda^{2} e^{\lambda \xi}}{1+\lambda d e^{\lambda \xi}}\right)+\frac{1}{2} C_{3} \lambda T \\
& +\frac{c C_{3}}{4 k_{2} C_{1} k_{1}} Y+C_{5},
\end{aligned}
$$

where $\xi=C_{1} X+C_{2}$.

$$
\begin{aligned}
u_{2}(x, y, t)= & -2 C_{1} k_{1}\left(\frac{d \lambda^{2} e^{\lambda \xi}}{1+\lambda d e^{\lambda \xi}}\right)+\frac{1}{2} C_{1} k_{1} \lambda^{2} F(Y) \\
& +C_{2}
\end{aligned}
$$

where $\xi=C_{1} X+F(Y)$.

By a combination of (18) and the results above, one can obtain the following solitary wave solutions:

$$
\begin{aligned}
u_{3}(x, y, t)= & -\frac{\lambda}{2}\left(C_{3} T+C_{4}\right)\left[1+\tanh \left(\frac{\lambda \xi}{2}\right)\right] \\
& +\frac{1}{2} C_{3} \lambda T+\frac{c C_{3}}{4 k_{2} C_{1} k_{1}} Y+C_{5},
\end{aligned}
$$

where $\xi=C_{1} X+C_{2}$.

$$
\begin{aligned}
u_{4}(x, y, t)= & -C_{1} k_{1} \lambda\left[1+\tanh \left(\frac{\lambda \xi}{2}\right)\right] \\
& +\frac{1}{2} C_{1} k_{1} \lambda^{2} F(Y)+C_{2},
\end{aligned}
$$

where $\xi=C_{1} X+F(Y)$

Case 2. If $\psi(\xi)$ satisfies (29), then substituting (44) into (42), using (29), collecting all the terms with the same power of $\psi$ together, and equating each coefficient to zero yield a set of underdetermined partial differential equations. Solving these equations yields the following results. 
Family 1

$$
\begin{aligned}
a_{0}(Y, T) & =2 C_{1} k_{1} \lambda^{2} F_{2}(Y)+C_{2}, \\
a_{1}(Y, T) & =4 C_{1} k_{1}, \\
\xi(X, Y, T) & =C_{1} X+F_{2}(Y) .
\end{aligned}
$$

Family 2

$$
\begin{aligned}
a_{0}(Y, T) & =\frac{1}{2} C_{3} \lambda T+\frac{c C_{3}}{8 k_{2} C_{1} k_{1}} Y+C_{5}, \\
a_{1}(Y, T) & =C_{3} T+C_{4}, \\
\xi(x, y, T) & =C_{1} X+C_{2} .
\end{aligned}
$$

By a combination of the results above and (35), one can obtain the following exact solutions for (41), where $T=$ $c\left(t^{\alpha} / \alpha\right), X=k_{1}\left(x^{\alpha} / \alpha\right), Y=k_{1}\left(y^{\alpha} / \alpha\right)$.

$$
\begin{aligned}
u_{5}(x, y, t)= & -4 C_{1} k_{1}\left(\frac{d \lambda^{2} e^{\lambda \xi}}{1+\lambda d e^{\lambda \xi}}\right)+2 C_{1} k_{1} \lambda^{2} F_{2}(Y) \\
& +C_{2}
\end{aligned}
$$

where $\xi=C_{1} X+F_{2}(Y)$

$$
\begin{aligned}
u_{6}(x, y, t)= & -\left(C_{3} T+C_{4}\right)\left(\frac{d \lambda^{2} e^{\lambda \xi}}{1+\lambda d e^{\lambda \xi}}\right)+\frac{1}{2} C_{3} \lambda T \\
& +\frac{c C_{3}}{8 k_{2} C_{1} k_{1}} Y+C_{5},
\end{aligned}
$$

where $\xi=C_{1} X+C_{2}$.

Similarly, by a combination of (40) and the results above, one can obtain corresponding solitary wave solutions, which are omitted here.

\section{Conclusions}

Based on the properties of conformable fractional calculus, we have proposed an auxiliary equation method for seeking exact solutions with variable coefficient function forms for fractional partial differential equations and applied it to the time fractional two-dimensional Boussinesq equation and the space-time fractional $(2+1)$-dimensional breaking soliton equation. As a result, some exact solutions including variable coefficient function solutions as well as solitary wave solutions for them have been successfully found. These solutions are new exact solutions so far in the literature to the best of our knowledge. We note that, by a combination of other auxiliary equations different from the two equations used here, more exact solutions can be found subsequently.

\section{Data Availability}

The data used to support the findings of this study are included within the article.

\section{Conflicts of Interest}

The authors declare that there are no conflicts of interest regarding the publication of this paper.

\section{Acknowledgments}

This work was partially supported by Natural Science Foundation of China (11671227) and the development supporting plan for young teachers in Shandong University of Technology.

\section{References}

[1] Q. Feng and F. Meng, "Oscillation of solutions to nonlinear forced fractional differential equations," Electronic Journal of Differential Equations, vol. 169, pp. 1-10, 2013.

[2] J. Shao, Z. Zheng, and F. Meng, "Oscillation criteria for fractional differential equations with mixed nonlinearities," Advances in Difference Equations, vol. 323, pp. 1-9, 2013.

[3] L. Guo, L. Liu, and Y. Wu, "Existence of positive solutions for singular fractional differential equations with infinite-point boundary conditions," Nonlinear Analysis, Modelling and Control, vol. 21, no. 5, pp. 635-650, 2016.

[4] L. Ren and J. Xin, "Almost global existence for the Neumann problem of quasilinear wave equations outside star-shaped domains in 3D," Electronic Journal of Differential Equations, vol. 312, pp. 1-22, 2017.

[5] Y. Sun, L. Liu, and Y. Wu, "The existence and uniqueness of positive monotone solutions for a class of nonlinear Schrödinger equations on infinite domains," Journal of Computational and Applied Mathematics, vol. 321, pp. 478-486, 2017.

[6] Q. Feng and F. Meng, "Finite difference scheme with spatial fourth-order accuracy for a class of time fractional parabolic equations with variable coefficient," Advances in Difference Equations, vol. 305, pp. 1-14, 2016.

[7] O. Unsal, O. Guner, and A. Bekir, "Analytical approach for space-time fractional Klein-Gordon equation," Optik - International Journal for Light and Electron Optics, vol. 135, pp. 337-345, 2017.

[8] B. Agheli, R. Darzi, and A. Dabbaghian, "Computing exact solutions for conformable time fractional generalized seventhorder KdV equation by using $\left(G^{\prime} / G\right)$-expansion method," Optical and Quantum Electronics, vol. 49, no. 387, pp. 1-13, 2017.

[9] B. Zheng, "Exact solutions for some fractional partial differential equations by the $\left(G^{\prime} / G\right)$ method," Mathematical Problems in Engineering, vol. 2013, Article ID 826369, 13 pages, 2013.

[10] T. Islam, M. A. Akbar, and A. K. Azad, "Traveling wave solutions to some nonlinear fractional partial differential equations through the rational $\left(G^{\prime} / G\right)$-expansion method," Journal of Ocean Engineering and Science, vol. 3, no. 1, pp. 76-81, 2018.

[11] B. Zheng and Q. Feng, "A New Approach for Solving Fractional Partial Differential Equations in the Sense of the Modified Riemann-Liouville Derivative," Mathematical Problems in Engineering, vol. 2014, Article ID 307371, 7 pages, 2014.

[12] H. Rezazadeh, A. Korkmaz, M. Eslami, J. Vahidi, and R. Asghari, "Traveling wave solution of conformable fractional generalized reaction Duffing model by generalized projective Riccati equation method," Optical and Quantum Electronics, vol. 50, no. 150, pp. 1-13, 2018.

[13] A. Korkmaz, "Exact solutions to $(3+1)$ conformable time fractional Jimbo-Miwa, Zakharov-Kuznetsov and modified Zakharov-Kuznetsov equations," Communications in Theoretical Physics, vol. 67, no. 5, pp. 479-482, 2017. 
[14] D. Kumar, A. R. Seadawy, and A. K. Joardar, "Modified Kudryashov method via new exact solutions for some conformable fractional differential equations arising in mathematical biology," Chinese Journal of Physics, vol. 56, no. 1, pp. 75-85, 2018.

[15] K. Hosseini, A. Bekir, and R. Ansari, "New exact solutions of the conformable time-fractional Cahn-Allen and Cahn-Hilliard equations using the modified Kudryashov method," Optik International Journal for Light and Electron Optics, vol. 132, pp. 203-209, 2017.

[16] K. Hosseini, P. Mayeli, and R. Ansari, "Modified Kudryashov method for solving the conformable time-fractional KleinGordon equations with quadratic and cubic nonlinearities," Optik - International Journal for Light and Electron Optics, vol. 130, pp. 737-742, 2017.

[17] K. Hosseini, F. Samadani, D. Kumar, and M. Faridi, "New optical solitons of cubic-quartic nonlinear Schrödinger equation," Optik - International Journal for Light and Electron Optics, vol. 157, pp. 1101-1105, 2018.

[18] K. Hosseini, D. Kumar, M. Kaplan, and E. Y. Bejarbaneh, "New Exact Traveling Wave Solutions of the Unstable Nonlinear Schrödinger Equations," Communications in Theoretical Physics, vol. 68, no. 6, pp. 761-767, 2017.

[19] M. Kaplan, K. Hosseini, F. Samadani, and N. Raza, "Optical soliton solutions of the cubic-quintic non-linear Schrödinger's equation including an anti-cubic term," Journal of Modern Optics, vol. 65, no. 12, pp. 1431-1436, 2018.

[20] A. Korkmaz and K. Hosseini, "Exact solutions of a nonlinear conformable time-fractional parabolic equation with exponential nonlinearity using reliable methods," Optical and Quantum Electronics, vol. 49, no. 278, pp. 1-10, 2017.

[21] M. Lakestani and J. Manafian, "Analytical treatment of nonlinear conformable time-fractional Boussinesq equations by three integration methods," Optical and Quantum Electronics, vol. 50, no. 4, pp. 1-31, 2018.

[22] K. Hosseini, A. Bekir, M. Kaplan, and Ö. Güner, "On a new technique for solving the nonlinear conformable time-fractional differential equations," Optical and Quantum Electronics, vol. 49, no. 343, pp. 1-12, 2017.

[23] K. Hosseini, P. Mayeli, A. Bekir, and O. Guner, "DensityDependent Conformable Space-time Fractional DiffusionReaction Equation and Its Exact Solutions," Communications in Theoretical Physics, vol. 69, pp. 1-4, 2018.

[24] K. Hosseini, Y.-J. Xu, P. Mayeli et al., "A study on the conformable time-fractional Klein-Gordon equations with quadratic and cubic nonlinearities," Optoelectronics and Advanced Materials - Rapid Communications, vol. 11, pp. 423-429, 2017.

[25] K. Hosseini, J. Manafian, F. Samadani, M. Foroutan, M. Mirzazadeh, and Q. Zhou, "Resonant optical solitons with perturbation terms andfractional temporal evolution using improved $\tan (\Phi(\eta) / 2)$-expansion method and exp function approach," Optik - International Journal for Light and Electron Optics, vol. 158, pp. 933-939, 2018.

[26] K. Hosseini, P. Mayeli, and R. Ansari, "Bright and singular soliton solutions of the conformable time-fractional Klein-Gordon equations with different nonlinearities," Waves in Random and Complex Media: Propagation, Scattering and Imaging, vol. 28, no. 3, pp. 426-434, 2018.

[27] Y. Çenesiz, D. Baleanu, A. Kurt, and O. Tasbozan, "New exact solutions of Burgers' type equations with conformable derivative," Waves in Random And Complex Media: Propagation, Scattering and Imaging, vol. 27, no. 1, pp. 103-116, 2017.

[28] M. Eslami, F. S. Khodadad, F. Nazari, and H. Rezazadeh, “The first integral method applied to the Bogoyavlenskii equations by means of conformable fractional derivative," Optical and Quantum Electronics, vol. 49, no. 12, 2017.

[29] M. Ekici, M. Mirzazadeh, M. Eslami et al., "Optical soliton perturbation with fractional-temporal evolution by first integral method with conformable fractional derivatives," Optik - International Journal for Light and Electron Optics, vol. 127, no. 22, pp. 10659-10669, 2016.

[30] F. Meng and Q. Feng, "A new fractional subequation method and its applications for space-time fractional partial differential equations," Journal of Applied Mathematics, vol. 2013, 10 pages, 2013.

[31] F. Meng, "A new approach for solving fractional partial differential equations," Journal of Applied Mathematics, vol. 2013, Article ID 256823, 5 pages, 2013.

[32] I. Aslan, "Traveling wave solutions for nonlinear differentialdifference equations of rational types," Communications in Theoretical Physics, vol. 65, no. 1, pp. 39-45, 2016.

[33] Q. Feng and F. Meng, "Explicit solutions for space-time fractional partial differential equations in mathematical physics by a new generalized fractional Jacobi elliptic equation-based subequation method," Optik - International Journal for Light and Electron Optics, vol. 127, no. 19, pp. 7450-7458, 2016.

[34] Q. Feng and F. Meng, "Traveling wave solutions for fractional partial differential equations arising in mathematical physics by an improved fractional Jacobi elliptic equation method," Mathematical Methods in the Applied Sciences, vol. 40, no. 10, pp. 3676-3686, 2017.

[35] R. Khalil, M. Al Horani, A. Yousef, and M. Sababheh, "A new definition of fractional derivative," Journal of Computational and Applied Mathematics, vol. 264, pp. 65-70, 2014.

[36] O. Tasbozan, Y. Çenesiz, and A. Kurt, "New solutions for conformable fractional Boussinesq and combined KdV-mKdV equations using Jacobi elliptic function expansion method," The European Physical Journal Plus, vol. 131, no. 244, pp. 1-14, 2016.

[37] M. M. Khater and D. Kumar, "Implementation of three reliable methods for finding the exact solutions of $(2+1)$ dimensional generalized fractional evolution equations," Optical and Quantum Electronics, vol. 49, no. 427, pp. 1-16, 2017. 


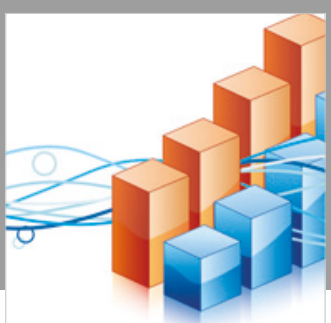

Advances in

Operations Research

\section{-n-m}
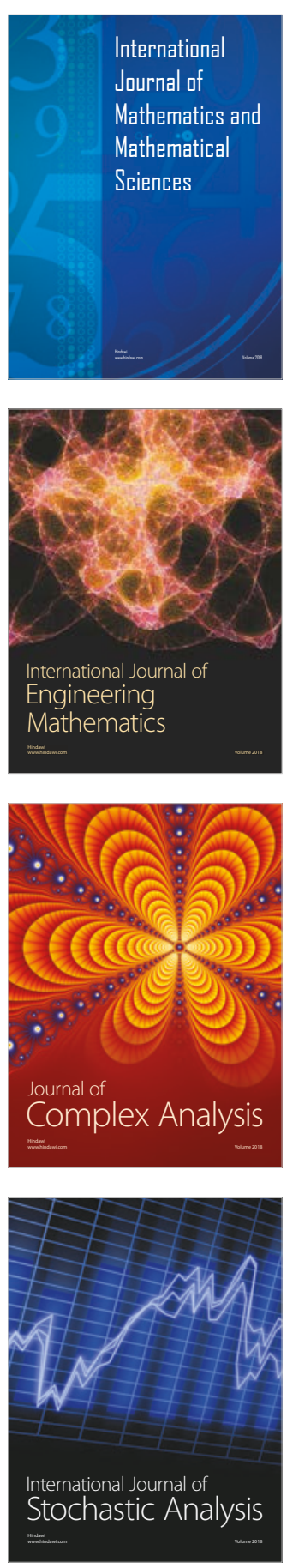
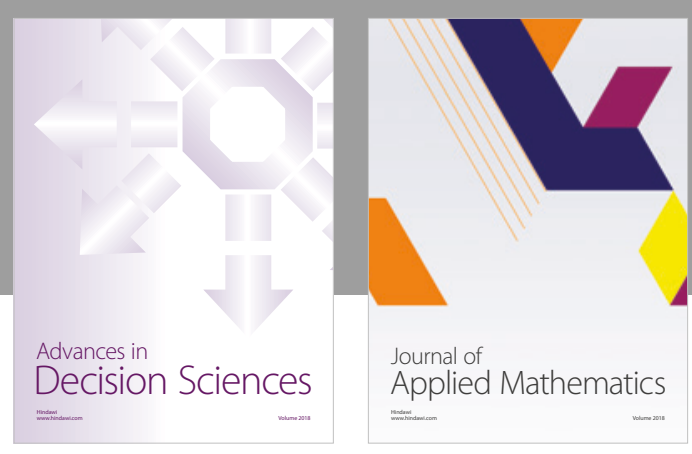

Journal of

Applied Mathematics
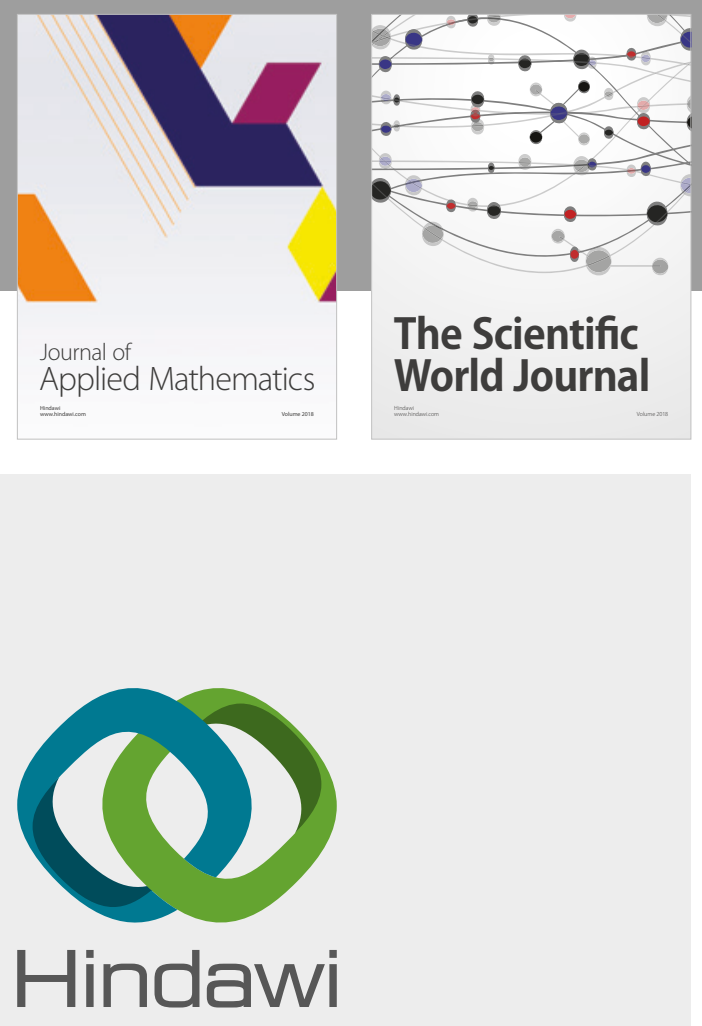

Submit your manuscripts at

www.hindawi.com

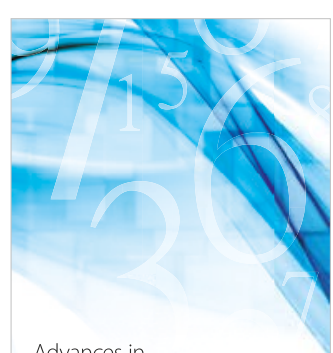

Advances in
Numerical Analysis
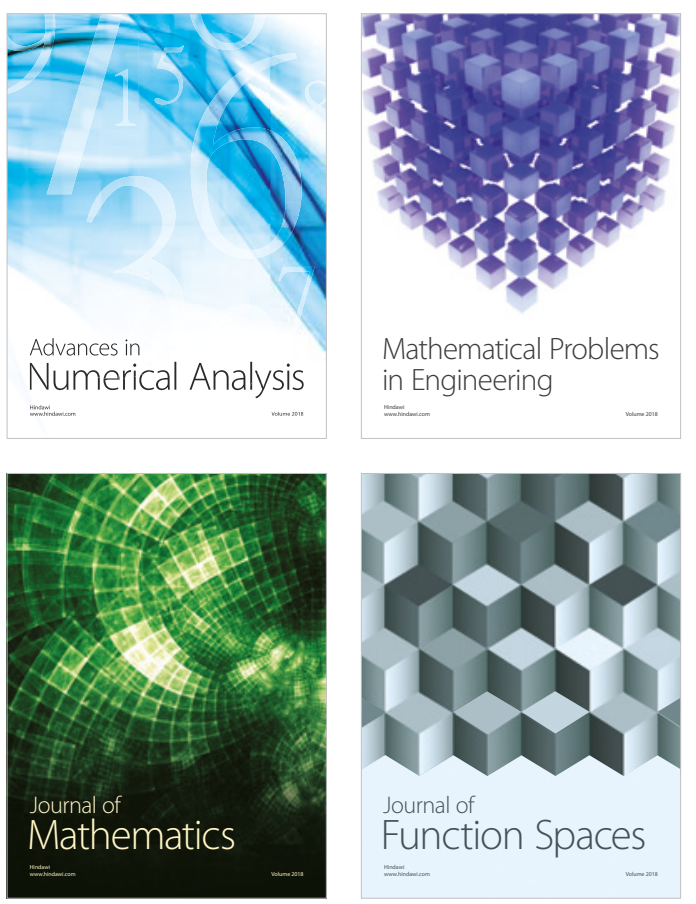

Mathematical Problems in Engineering

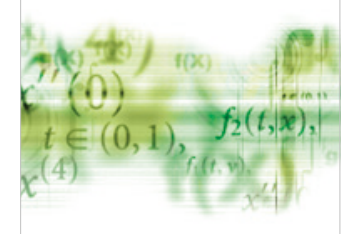

International Journal of

Differential Equations

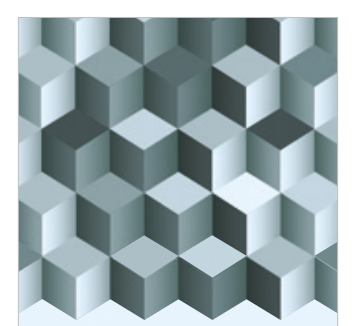

Journal of

Function Spaces

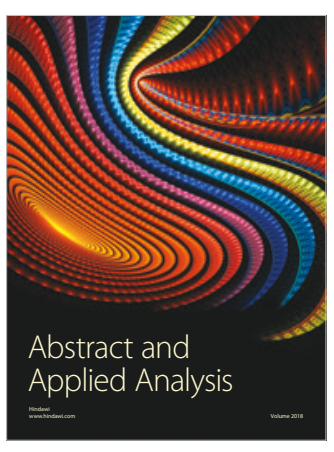

The Scientific

World Journal

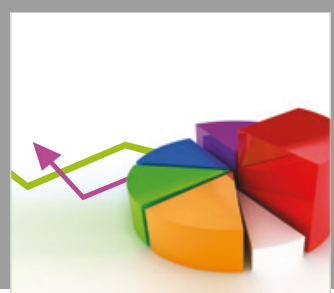

Journal of

Probability and Statistics
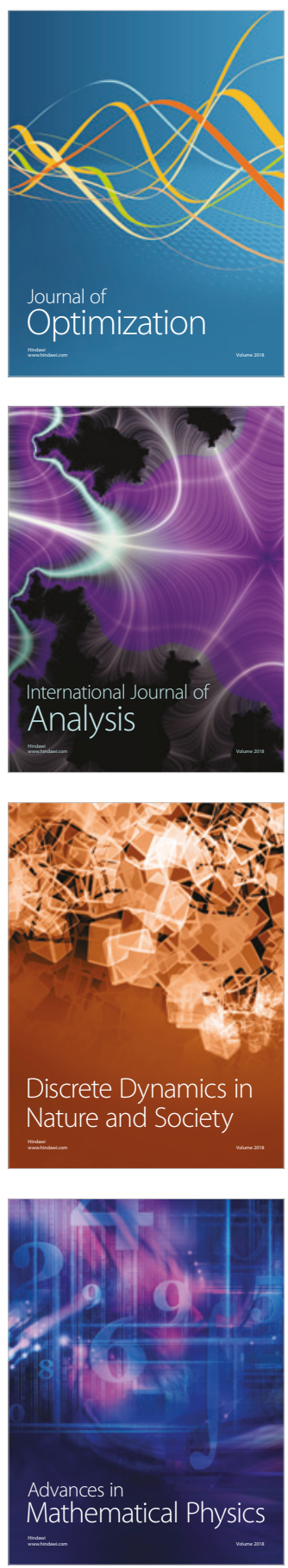